\title{
Percepciones sobre el uso de bases de datos en investigación formativa en odontología
}

\section{Perception on the use of databases in training research in dentistry}

\section{Percepções sobre o uso de bancos de dados na pesquisa de treinamento em odontologia}

Lilia Jadith Bernal-Cepeda $1 \underline{\underline{\mathrm{ORCID}}}$, Jesús Armando Tobar-Sánchez $2 \underline{\underline{\mathrm{ORCID}}}$, Margarita María Misas-Avella ${ }^{3 凶} \underline{\text { ORCID }}$

\footnotetext{
${ }^{1}$ Odontóloga / Magíster en Ciencias-Farmacología / Especialista en Docencia Universitaria / Magíster en Docencia de la Educación Superior / cPhD Ciencias-Farmacéuticas. Profesor asistente, Facultad de Odontología Universidad Nacional de Colombia; Profesor asistente, Facultad de Odontología Universidad El Bosque; Bogotá-Colombia.

2 Ingeniero Químico/Especialista en Docencia Universitaria. Profesor auxiliar, Facultad de Estudios Técnicos y Tecnológicos, Universidad Autónoma de Bucaramanga.

${ }^{3}$ Literata / Especialista en Docencia Universitaria / Magíster en Educación / Candidata a Magíster en Estudios Sociales y Culturales. Docente del Instituto de Educación a Distancia de la Universidad del Tolima. Docente del Instituto Pedagógico Nacional, de la Universidad Pedagógica Nacional.
}

Fecha correspondencia:

Recibido: octubre de 2020

Aprobado: septiembre de 2021.

Forma de citar:

Bernal-Cepeda LJ, Tobar-Sánchez JA, Misas-Avella MM.

Percepciones sobre el uso de bases de datos en investigación formativa en odontología. Rev. CES Odont 2021; 34(2): 76-92. https://doi.org/10.21615/ cesodon.5925

Open access

(C) Derecho de autor

Licencia creative commons

Ética de publicaciones

Revisión por pares

Gestión por Open Journal System

\section{Resumen}

Actualmente existen varias estrategias para consultar información en la red en tiempo real sobre infinidad de temas, tanto asuntos de la cotidianidad de las personas como de campos del saber que son de interés para la comunidad académica. Las bases de datos se constituyen como herramientas eficientes para la trasmisión de información y su acceso, en general, resulta sencillo y rápido; sin embargo, en ocasiones estudiantes y docentes no las emplean con la frecuencia que se desearía, fenómeno del que se desconocen en gran medida las causas. El objetivo del presente trabajo fue indagar las percepciones de docentes y estudiantes de una Facultad de Odontología en Bogotá, en cuanto al uso de las bases de datos en investigación formativa, empleando la técnica de Clasificación Múltiple de Ítems -CMI-. Se pudo identificar que para la mayoría de los participantes se hace necesario implementar estrategias pedagógicas que promuevan el uso de estas; adicionalmente los participantes reconocen la importancia 
DOI: 10.21615/cesodon.5925

ISSNe 2215-9185

ISSN 0120-971X

Publica con nosotros de los diferentes actores en el proceso enseñanza-aprendizaje y de la inclusión de las bases de datos en las diferentes actividades académicas e investigativas.

Palabras clave: base de datos; conocimiento; educación; investigación; Odontología.

\section{Abstract}

Nowadays, many strategies are available to search for information on the web in real-time about various topics, including personal daily life and academic knowledge, which are of interest to the academic community. Databases are efficient tools for transmitting and accessing to information in an easy and quick manner. Nevertheless, students and faculty frequently do not take advantage of it as expected, whose cause is unknown.

The aim of this work was asked the faculty and students of the Dentistry Department of an University of Bogota about the perceptions on the use of databases in training research, employing the Multiple Classification of Items -MCl-. We identified that for most of the participants it is necessary to implement pedagogical strategies that promotes the use of these; additionally, the participants recognize the importance of the different members in the teaching-learning process and the database inclusion in the different academic and research activities.

Keywords: databases; knowledge; education; research; dentistry.

\section{Resumo}

Atualmente, existem várias estratégias para consultar informações em tempo real na web sobre uma infinidade de assuntos, tanto no cotidiano das pessoas quanto em áreas do conhecimento de interesse da comunidade acadêmica. As bases de dados constituem-se em ferramentas eficientes para a transmissão de informações e o seu acesso, em geral, é simples e rápido; no entanto, às vezes os alunos e professores não os usam com a freqüência desejada, um fenômeno cujas causas são amplamente desconhecidas.

O objetivo deste trabalho foi investigar as percepções de professores e alunos de uma Faculdade de Odontologia de Bogotá, a respeito da utilização de bancos de dados em pesquisas formativas, utilizando a técnica de Classificação de Múltiplos Itens -CMI-. Foi possível identificar que para a maioria dos participantes é necessário implementar estratégias pedagógicas que 
promovam o uso destes; Além disso, os participantes reconhecem a importância dos diferentes atores no processo de ensino-aprendizagem e na inclusão de bases de dados nas diferentes atividades acadêmicas e de pesquisa.

Palavras-chave: bancos de dados; conhecimento; educação; pesquisa; odontologia.

\section{Introducción}

El desarrollo de la tecnología ha contribuido de manera significativa al avance de la educación $y$, dentro de ella, las bases de datos se presentan como herramientas de uso sencillo, económicas y confiables para lograr el objetivo de acceder al conocimiento, favoreciendo el mejoramiento del ejercicio académico y profesional. La disponibilidad de esta información virtual ha tenido un fuerte impacto sobre la educación, siendo uno de los mayores cambios en las instituciones educativas en las últimas décadas ${ }^{(1)}$.

Una base de datos puede definirse como un conjunto de información sistemáticamente almacenada que posee un contexto similar y que puede consultarse de manera rápida y sencilla cuando así se requiera ${ }^{(2)}$. Adicionalmente el empleo de redes de comunicación minimiza las barreras geográficas y temporales.

Son múltiples las voces que señalan con insistencia que la educación se favorece con la consulta y análisis de información que se encuentra disponible virtualmente, por ejemplo, en la Primera y Segunda Conferencia Mundial sobre la Educación Superior, se sugirió que las nuevas tecnologías de la información desempeñan un papel preponderante en el acceso al conocimiento y en el avance de la educación superior ${ }^{(3)}$, además de hacer un llamado a promover el uso de las tecnologías de la información y las comunicaciones (TIC) en el acceso a educación de calidad ${ }^{(4)}$. Por otra parte, en Latinoamérica el Proyecto Tuning dentro de las competencias generales, describe la necesidad del desarrollo de "habilidades en el uso de las tecnologías de la información y de la comunicación [...] y habilidades para buscar, procesar y analizar información procedente de fuentes diversas" ${ }^{(5)}$.

En el campo de la Odontología dentro de los perfiles y competencias de los profesionales establecidos por el Ministerio de Salud y Protección Social (2016), se destaca el fortalecimiento de las capacidades comunicativas e investigativas, y se plantean criterios de desempeño como "interpreta textos científicos con sentido crítico para resolver problemas relacionados con el ejercicio profesional [y] participa en el diseño e implementación de sistemas de información con base en referentes nacionales e internacionales" (6). 
A pesar de lo descrito anteriormente, la sola existencia de las bases de datos y la declaración de buenas intenciones por parte de organizaciones multilaterales e instituciones educativas no puede garantizar el empleo de las mismas. Algunos autores reportan que es necesario el entrenamiento sobre estas herramientas, con el objetivo de fomentar el desarrollo académico y favorecer de otro modo la producción científica ${ }^{(7)}$.

De acuerdo con lo planteado en el presente trabajo, resulta importante diferenciar los términos de percepción y opinión. El primero consiste en un proceso activo, que resulta del procesamiento de la información obtenida del entorno y la organización a través de juicios categoriales, para poder brindar una buena representación a los objetos en cuestión ${ }^{(8)}$. Por su parte, la opinión consiste en el juicio o concepto que un individuo tiene de determinado elemento.

En consecuencia, el objetivo del presente estudio fue, conocer cuáles son las percepciones que poseen los diferentes miembros de la comunidad educativa en relación con el empleo de las bases de datos durante su formación académica, para identificar los posibles problemas que se presentan, y con lo cual se podría mejorar en el proceso de introducción de estas herramientas en las actividades diarias.

\section{Materiales y métodos}

Este trabajo fue desarrollado dentro del paradigma cualitativo -bajo un enfoque interpretativohermenéutico-. Como mencionan Hernández Sampieri et al (2010): “La investigación cualitativa se fundamenta en una perspectiva interpretativa centrada en el entendimiento del significado de las acciones de seres vivos, sobre todo de los humanos y sus instituciones ${ }^{(9)}$. Los investigadores describen las percepciones obtenidas de los participantes, acerca del grado de conocimiento $y$, sobre todo, el uso que hacen de las bases de datos en la investigación formativa. Posteriormente se realiza un análisis de dichas percepciones para establecer algunas recomendaciones que incentiven el uso adecuado de las bases de datos en el ámbito universitario.

La técnica empleada fue $\mathrm{CMI}$ que es "un tipo de entrevista individual que se fundamenta en el surgimiento libre y espontáneo de criterios por parte del entrevistado para realizar clasificaciones cualitativas sobre un tema en particular" ${ }^{(10)}$. Se eligieron 22 ítems con los cuales se construyeron igual número de tarjetas cuadradas de $7 \mathrm{~cm}$ de lado, que incluían el nombre del ítem, así como una imagen superior relacionada con la palabra; cada ítem recibió un código para facilitar el procesamiento de la información (Tabla 1). 
Tabla 1. Codificación de los ítems seleccionados para la CMI. Elaboración propia.

\begin{tabular}{cccccc}
\hline Código & Ítem & Código & Ítem & Código & Ítem \\
\hline 1 & Base de datos & 9 & Debilidad & 16 & Capacitación \\
\hline 2 & $\begin{array}{c}\text { Información } \\
\text { científica }\end{array}$ & 10 & Transmisión & 17 & Aprendizaje \\
\hline 3 & Frecuencia & 11 & Confiabilidad & 18 & $\begin{array}{c}\text { Aprendizaje } \\
\text { significativo }\end{array}$ \\
\hline 4 & Utilidad & 12 & Educación & 19 & \begin{tabular}{c} 
Odontología \\
\hline 5
\end{tabular} \\
Actualización & 13 & Enseñanza & 20 & $\begin{array}{c}\text { Universidad El } \\
\text { Bosque }\end{array}$ \\
\hline 6 & Accesibilidad & 14 & $\begin{array}{c}\text { Estrategias } \\
\text { pedagógicas }\end{array}$ & 21 & Rol docente \\
\hline 7 & Globalización & 15 & Investigación & 22 & Rol estudiante \\
\hline 8 & Fortaleza & & & & \\
\hline
\end{tabular}

Como población de estudio se eligieron cuatro estudiantes (de quinto a octavo semestre) y cuatro docentes de la Facultad de Odontología de la Universidad El Bosque; dos de los estudiantes pertenecían a un semillero de investigación y dos no hacían parte de estos grupos, para de esta manera poder contrastar la información.

Respecto a los profesores, se seleccionaron dos docentes que tuvieran a su cargo asignaturas de investigación y dos que dirigieran semilleros de investigación, con el propósito de comparar percepciones de quienes trabajan fundamentos de investigación en el aula y los que se encontraban inmersos en ejercicios investigativos.

Los participantes fueron designados con los códigos P1, P2, P3, P4, P5, P6, P7 y P8 y se clasificaron en tres grupos: estudiantes que participaban en semillero de investigación (P1 y P2), estudiantes que no pertenecían a semilleros de investigación (P3 y P4) y docentes (P5, P6, P7 y $\mathrm{P} 8$ ).

Previo aval del comité técnico científico de la Facultad y diligenciamiento del consentimiento informado, donde se solicitaba autorización para grabar y se explicaba el objetivo de la investigación, se aplicó la técnica, en la cual se orientó al entrevistado para que realizara el trabajo de clasificación, buscando a su vez la reducción de sesgos del investigador y del participante. Al observar la tarjeta, el entrevistado empleaba sus habilidades semánticas (el sentido del título de cada tarjeta), las semióticas (relacionar la imagen con el concepto que se 
le presentó) y las pragmáticas (teniendo en cuenta el contexto comunicativo), para la generación del proceso de asociación ${ }^{(11)}$.

Durante la realización de la $\mathrm{CMI}$, se generó un registro fílmico de las manos y la voz, para el diligenciamiento del formato de almacenamiento de información que contenía criterio de clasificación, subcategoría, tarjetas de la subcategoría y observaciones establecidas por cada participante (Tabla 2). Los participantes contaban con plena libertad de realizar el número de clasificaciones que consideraran pertinentes.

Tabla 2. Formato de obtención de datos. Basada en Páramo (2008).

\begin{tabular}{|c|c|c|c|}
\hline Criterio & Subcategoría & $\begin{array}{l}\text { Tarjetas de la } \\
\text { subcategoría }\end{array}$ & $\begin{array}{l}\text { Observaciones } \\
\text { del participante }\end{array}$ \\
\hline & & & \\
\hline & & & \\
\hline
\end{tabular}

Posterior a la recolección y organización de la información en un formato de Microsoft Excel ${ }^{\mathrm{TM}}$, se realizó la generación de nodos por asociación con el empleo del software Nvivo ${ }^{T M}$ (versión 11), que permite el análisis de las perspectivas de datos no estructurados o cualitativos como entrevistas y respuestas de encuestas con preguntas abiertas ${ }^{(12)}$. Los datos introducidos fueron los nombres de los ítems y los valores numéricos para codificar la subcategoría en la cual cada participante incluyó las diferentes tarjetas. Estos valores numéricos no implican la realización de análisis cuantitativo, debido a que en estas condiciones el número solo es un atributo que permite correlacionar la información y su organización. Se generaron 4 nodos conglomerados por asociación: uno por valor de atributo para P1 y P2, otro para los participantes 3 y 4 , un tercer nodo donde se incluyeron los resultados de los docentes y un nodo final donde se encontraban relacionados los resultados de todos los participantes.

Finalmente, a partir de las gráficas de nodos, las categorías establecidas y las voces de los participantes, se realizó la interpretación de la información. 


\section{Resultados}

\section{Categorías establecidas por los autores}

Estas categorías se utilizaron para el análisis de las agrupaciones realizadas por los participantes y dentro de ellas se encuentran: elementos esenciales en el proceso enseñanza-aprendizaje, características y actores importantes en la apropiación del conocimiento, integración odontología e investigación, estrategias pedagógicas en la generación del aprendizaje significativo y características del proceso investigativo.

\section{Percepciones de los participantes}

Respecto al número de clasificaciones realizadas, P2, P3, P4 y P5 elaboraron una clasificación (Imagen 1), mientras que algunos participantes construyeron más de una, por ejemplo, P1 y P8 hicieron tres y $\mathrm{P} 6$ y $\mathrm{P7}$, dos clasificaciones.
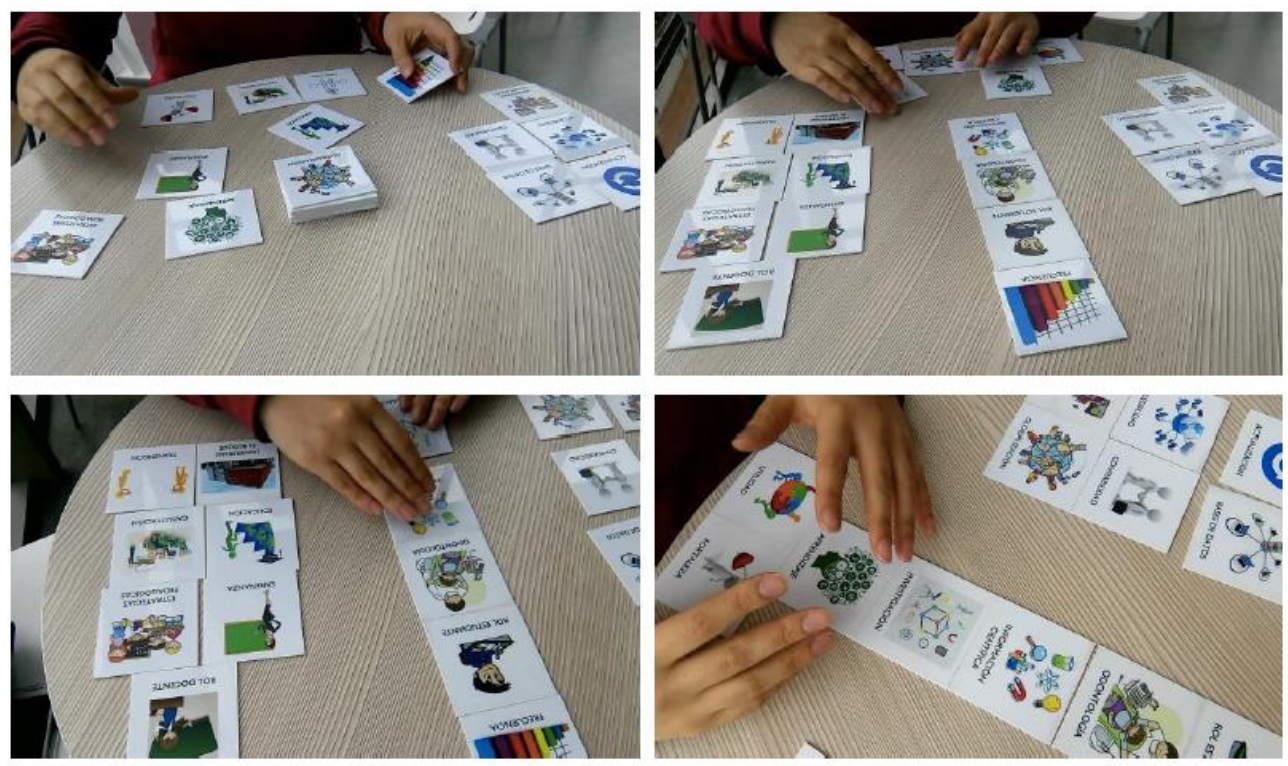

Imagen 1. Aplicación Clasificación Múltiple de Ítems: P2 realizando CMI. Elaboración propia. 


\section{Estudiantes que participaban en semilleros de investigación}

Las agrupaciones establecidas por el participante recibieron un nombre asignado por el mismo. P2, por ejemplo estableció "Institucional" y realizó tres subcategorías a las cuales denominó "lo que es importante para mí en la información" que contenía los ítems 1, 5, 6, 7, 11 y 18; "Mi parte como estudiante (mi aporte a mi capacitación)" donde se encontraban los elementos 2 , 3, 4, 8, 9 15, 17, 19 y 22; y "La parte institucional", donde incluyó las tarjetas 10, 12, 13, 14, 16, 20 y 21 . Estos datos fueron organizados en el formato, donde las tarjetas asignadas a la primera subcategoría recibieron el número 1, las de la segunda el número 2 y finalmente a las de la tercera subcategoría se les asignó el número 3 (Tabla 3); posteriormente se generó el nodo conglomerado por asociación.

Tabla 3. Matriz de resultados para P2. Elaboración propia.

\begin{tabular}{lllllllllllllllllllllll}
\hline Item & 1 & 2 & 3 & 4 & 5 & 6 & 7 & 8 & 9 & 10 & 11 & 12 & 13 & 14 & 15 & 16 & 17 & 18 & 19 & 20 & 21 & 22 \\
\hline $\boldsymbol{P 2}$ & 1 & 2 & 2 & 2 & 1 & 1 & 1 & 2 & 2 & 3 & 1 & 3 & 3 & 3 & 2 & 3 & 2 & 1 & 2 & 3 & 3 & 2 \\
\hline
\end{tabular}

Características y actores importantes en la apropiación del conocimiento

Para la descripción de esta agrupación, los estudiantes mencionaron "Desempeño docente en el proceso investigativo" y "mi aporte a mi capacitación". Al analizar las percepciones de este grupo, se encontró que los entrevistados reconocían la importancia de los diversos integrantes en el proceso enseñanza-aprendizaje y referían que "para adquirir procesos de investigación se requiere del rol docente que desempeña unas estrategias pedagógicas que deben ser claras, concisas y pertinentes [...] para que el estudiante esté en capacidad de avanzar, de conocer y crecer más en la educación" (P1).

Elementos esenciales en el proceso enseñanza-aprendizaje

En esta agrupación los estudiantes se refirieron a "Herramientas de investigación" y "Lo que es importante para mí en la información". Las voces de este grupo muestran que en el desarrollo de la investigación son indispensables diferentes elementos (incluidas las bases de datos científicas), que facilitan dicho proceso, como puede evidenciarse en la afirmación "en el proceso investigativo se va a requerir de unos aspectos, unas herramientas, donde vamos a encontrar bases de datos, en donde están incluidos diferentes estudios realizados por profesionales...que son útiles para la comunidad investigativa, académica y estudiantil"(P1). 
Se identificaron algunas falencias en las estrategias utilizadas para construir el conocimiento, porque a pesar de que se dispone de varias herramientas y de capacitaciones continuas, "Ia transmisión a veces no es la correcta" (P1), por lo cual se requiere el desarrollo de nuevas didácticas que permitan a los estudiantes evidenciar la aplicabilidad y las ventajas generadas por las bases de datos en su práctica diaria.

\section{Estudiantes que no participaban en semilleros de investigación}

Los estudiantes planteaban que la falta de tiempo por la carga académica pudiera constituirse en uno de los factores que afectan el proceso. También reconocen que requieren apropiarse de su conocimiento y ganar autonomía, procesos que mejorarían su desempeño académico y que tendría probablemente un impacto clínico positivo.

\section{Integración Odontología e investigación}

En esta clasificación los estudiantes señalaron las subcategorías "odontología", "grupo de estudio" y "herramientas", percibiendo la necesidad de una actualización permanente en Odontología como se evidencia en las voces "la información siempre está cambiando" (P3); esto coincidió con lo expresado por el otro integrante de este subgrupo "se necesita estar actualizado para la profesión" (P4).

Estrategias pedagógicas en la generación del aprendizaje significativo

En esta agrupación se hizo referencia a "enseñanza" y "debilidades". Estos participantes afirmaron que se requerían diversas estrategias porque los estudiantes son diferentes y su proceso de educabilidad es particular "hay gente que es muy visual, hay otras [sic] que son de escuchar, de escribir" (P3), reforzando la idea de que, aunque las bases de datos están disponibles, se requieren nuevas herramientas en el proceso de enseñabilidad y educabilidad.

\section{Percepciones de docentes}

Elementos esenciales en el proceso enseñanza-aprendizaje

En esta descripción los docentes señalaron "Entorno Universitario", "Características humanas", "Generación de nuevo conocimiento y actores" y "papel de la odontología". Se observó que los participantes reconocían la importancia de los diferentes miembros que intervienen en el proceso enseñanza-aprendizaje al afirmar que "el estudiante es el principal dentro del aprendizaje significativo [y] el docente se debe capacitar para hacer ese rol" (P8). 
Un elemento interesante fue observar cómo P6 y P7 ubicaron la tarjeta correspondiente al ítem Odontología en un grupo independiente y refirieron que "Odontología no sabría dónde ponerla" (P6) y que "[la odontología] es más un oficio, por eso lo dejé por fuera" (P7), mostrando que, a pesar de su labor docente, no logran generar asociación entre este ítem y los demás. Esto probablemente se encuentra ligado a la falta de conexión de la Odontología con el campo de las bases de datos, lo que genera dificultades en el aprendizaje significativo.

\section{Características del proceso investigativo}

En esta agrupación los docentes mencionaron "Eje principal", "Ventajas de usar información científica" y "formación académica". Para los participantes el acceso a la información científica por medio de las bases de datos es clave para el buen desempeño académico e investigativo de los estudiantes y para el proceso de actualización y globalización: "tener acceso a la información científica es muy importante para el proceso de investigación, para estar actualizados en los diferentes escenarios. [...] las bases de datos son muy importantes porque brindan todas esas herramientas" (P5), pues "a través de las bases de datos esto [el conocimiento] puede globalizarse, puede ser transmitido" (P7), ya que "la investigación es un eje central de la universidad" (P8).

Se identificaron algunos problemas en el proceso de uso frecuente de las bases de datos científicas, refiriendo que "los estudiantes no ven la aplicabilidad o la importancia que tiene acceder a las bases de datos para consultar información, entonces la frecuencia con que usan las bases de datos es baja, porque ellos tienen sus intereses dirigidos hacia la clínica [...] y se limitan a la información que les llega, mas no la buscan por sí mismos" (P5), lo cual se encuentra relacionado con la falta de aplicabilidad percibida por los estudiantes.

Los resultados obtenidos muestran que es importante que el estudiante gane autonomía en su proceso de aprendizaje, pero también que el docente genere nuevos caminos que faciliten a los estudiantes la apropiación del manejo de las bases de datos, ya que al encontrar una clara aplicabilidad aumenta la probabilidad de que las utilicen de manera frecuente, lo cual es relatado por P5, quien afirma "el acceso a bases de datos por parte de los estudiantes de Odontología es un poco obligado y lo hacen solamente en casos que sean estrictamente necesarios [...] quizás sea un problema de estrategias pedagógicas por las cuales los estudiantes no tienen un aprendizaje significativo" (P5) y se podría agregar que se requiere que "sea útil toda la información que se le da al estudiante y la pueda aplicar" (P8). 
En general, los participantes identificaron la disponibilidad de bases de datos en la Universidad. El acceso a estas herramientas hace parte del ámbito investigativo, el cual es un eje fundamental en la academia que se evidencia en la segunda partición del gráfico de nodo conglomerados por similitud, donde los ítems investigación, utilidad, accesibilidad, bases de datos, frecuencia e información científica se encuentran relacionados (Imagen 2).

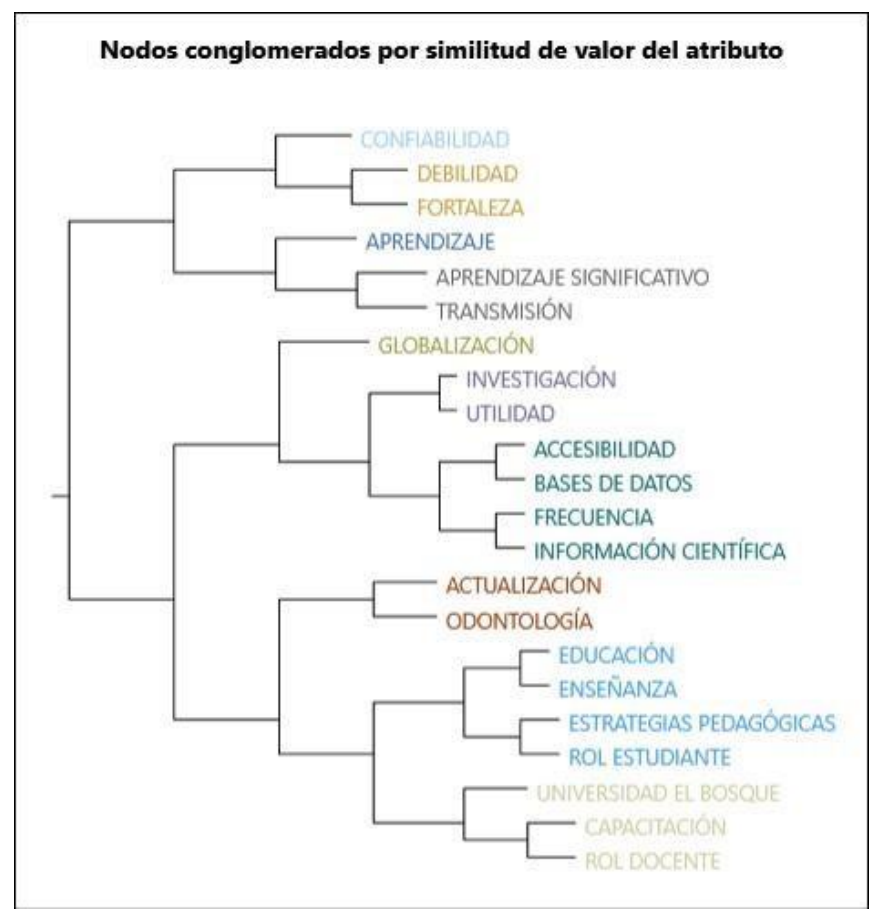

Imagen 2. Nodos conglomerados por valor de atributo para todos los participantes. Tomada de Nvivo ${ }^{\mathrm{TM}}$.

Del presente estudio se puede afirmar que los participantes: (a) Identificaron la importancia de la contribución de todos los actores (estudiantes y docentes) en el proceso enseñanzaaprendizaje y la implementación de las bases de datos en las diferentes actividades académicas e investigativas; (b) Reconocen que el uso de las bases de datos permitirá a los estudiantes y futuros profesionales estar en continua formación y actualización; (c) Describen la necesidad de que los docentes se capaciten en estas herramientas digitales, para que las empleen en su labor diaria e incentiven el empleo por parte de los estudiantes; (d) Refieren que se requiere la implementación de estrategias pedagógicas que promueva el empleo de las bases de datos durante la formación; (e) Reconocen que la Universidad hace un esfuerzo importante para que 
la comunidad académica tenga acceso a recursos confiables, actualizados y multidisciplinares, lo cual implica una inversión de recursos financieros, humanos y de tiempo; y (f) Analizan que es necesario que el estudiante tome un papel más activo y autónomo en la adquisición de habilidades referentes a la utilización de bases de datos.

\section{Discusión}

Desde hace varios años se ha hablado de los diferentes desafíos que enfrenta el mundo en el contexto actual: inquietudes, incertidumbres, dilemas, complejidades, posverdades, problemas científicos y académicos, entre otros. Por eso se requieren profesionales con alta capacitación para que puedan enfrentar y dar respuesta a estos interrogantes, siendo parte de los saberes fundamentales que la educación del futuro debería tratar en cualquier sociedad ${ }^{(13)}$. En este sentido la formación de los estudiantes que enfrentarán las situaciones anteriormente planteadas debe estar basada en hechos científicos y académicos de relevancia.

Los resultados obtenidos en el presente estudio mostraron que los participantes identificaron la importancia de implementar las bases de datos en las actividades académicas e investigativas para poder garantizar procesos exitosos. Tal y como mencionan Asenjo-Lobos y Manterola (2014) el desarrollo de la práctica clínica basada en la evidencia resulta fundamental, así como la búsqueda de literatura de forma sistemática y eficiente, y finalmente una lectura crítica para la evaluación objetiva de la información ${ }^{(14)}$.

Agudelo (2004) describe que las relaciones entre educación, comunicación y tecnología permiten el desarrollo de la autonomía y el pensamiento crítico en los estudiantes, similar a lo planteado en este estudio donde se resalta la importancia de estas cualidades para la consolidación del conocimiento ${ }^{(15)}$. En relación con esto, Cabero (2000) detalla que los entornos virtuales de formación deben propiciar el trabajo colaborativo entre los estudiantes además de potenciar la autonomía e independencia de estos, de esta manera deberán brindarse las herramientas para que el estudiante tenga la capacidad de elegir su ruta de aprendizaje ${ }^{(1)}$.

Los avances de la educación en las últimas décadas, mediados en parte por las TIC, han conllevado al cambio del papel del docente hacia facilitador de procesos, dado que el aprendizaje no solo se lleva a cabo en el aula de clase, sino también en otros ambientes de trabajo generados por el docente y los estudiantes, donde pueden emplearse estas herramientas digitales ${ }^{(16)}$. Esto ratifica que los docentes deben guiar la adquisición de conocimiento de manera innovadora, generando a su vez un aumento en el interés. 
El fortalecimiento de los entornos digitales, incluyendo la facilidad y la oportunidad en el acceso a las bases de datos, es decisivo en las circunstancias actuales que vive el mundo, ocasionadas por la pandemia de COVID-19. Bajo estos condicionamientos la migración de emergencia desde la presencialidad a la virtualidad, ha llevado a docentes e instituciones educativas a reinventarse y a introducir en sus clases elementos digitales que en algunas ocasiones se desconocían complemente.

Por su parte, los estudiantes se han visto en la necesidad de potenciar su interés por el empleo de dichas herramientas, incluidas las bases de datos. De esta manera, Cóndor-Herrera (2020) describe cómo Latinoamérica venía viviendo un estado de transición hacia la incursión en el campo de los entornos virtuales de aprendizaje (EVA), E-learning, M-learning, B-learning y las TIC; sin embargo, la mayoría de países de la región no estaban preparados para la completa virtualidad. El autor plantea que para el éxito en este contexto, se requieren tres acciones: "La implementación de recursos tecnológicos, capacitación del personal docente [y] adaptación de los estudiantes a esta forma de educación" (17).

En el campo de la Odontología, Lanchares (2000) relata la necesidad de virtualizar los contenidos teóricos de los programas a través de un máximo rendimiento de las TIC. Adicionalmente, describe que la pandemia corresponde a un escenario de incertidumbre que invita a la reinvención para asegurar una formación fluida y de calidad ${ }^{(18)}$.

Aunque las facultades generalmente brindan capacitaciones en los primeros semestres de formación sobre el empleo de bases de datos, se requiere que este proceso se desarrolle de manera longitudinal, dado que son los estudiantes vinculados a procesos investigativos quienes realizan un mayor empleo de estas. Esto es soportado por lo descrito por Romanov et al. (2006), quienes detallan que existe mayor consulta de bases de datos en el primer año de la formación universitaria, y que un porcentaje significativo no accede al texto completo de los artículos limitando la información obtenida al resumen, lo que pudiera deberse al desconocimiento de los mecanismos disponibles para obtener el texto completo o al desinterés de conocer la información a profundidad ${ }^{(19)}$.

La formación en investigación, sin duda alguna, implica la capacitación en las herramientas disponibles y en el manejo de la información, acorde con lo encontrado en este estudio donde se manifiesta el requerimiento de capacitación docente en el campo disciplinar y pedagógico. De esta manera, Barros y Turpo (2017), describen la necesidad de capacitar al docente desde el ámbito personal e institucional en investigación, con el objetivo de que puedan participar en 
el avance de la educación superior ${ }^{(20)}$. Infante et al (2014), mediante la realización de cuestionarios a profesores de una institución universitaria, determinaron el bajo nivel de manejo de bases de datos electrónicas, por lo cual sugieren la realización de actualizaciones a través de formación continua ${ }^{(21)}$.

A nivel Colombia, Franco (2015) analizó las vivencias asociadas al proceso de investigación formativa de los estudiantes de Odontología de la Universidad de Antioquia (Medellín), así como las reflexiones generadas durante este proceso. El autor describe que uno de los inconvenientes que se encuentra durante la fase de formación, es la falta de estrategias pedagógicas: "Hacer docencia investigativa requiere un esfuerzo pedagógico y didáctico que, en muchas ocasiones, los profesores no están dispuestos a asumir o, cuando lo están, no reciben apoyo de los administradores" (22).

De manera similar, Córdoba (2016) puntualiza que para los docentes los procesos investigativos requieren esfuerzos adicionales ${ }^{(23)}$. Estos reportes junto con los resultados obtenidos en este trabajo muestran que para llevar a cabo esta transformación se requiere del compromiso de todos los miembros que participan en la interacción enseñabilidad-educabilidad.

Finalmente, se desea proponer una estrategia en la cual el docente actúe como generador de condiciones extrínsecas que creen motivación intrínseca: es importante que el docente desempeñe un papel activo en la concientización sobre la importancia del uso de las bases de datos en la formación académica e investigativa, de la mano con las necesidades planteadas por algunos autores, quienes describen la insuficiencia en la articulación entre docencia e investigación. El docente, independientemente de la asignatura, debería incorporar las bases de datos dentro de su estructura pedagógica y sus didácticas como ejes articuladores del aprendizaje, a través, por ejemplo, del planteamiento de situaciones problema que conduzcan a los estudiantes a la búsqueda y análisis de información científica. El estudiante por su parte debe ser receptivo frente a las estrategias planteadas y esto se podría obtener si logra comprender los beneficios que puede alcanzar en el desempeño clínico y académico, además de permitir la articulación entre la teoría y la práctica.

\section{Conclusiones}

Los participantes del estudio reconocen la importancia de la inclusión de las bases de datos en sus actividades académicas e investigativas, además de la necesidad de la participación activa de todos los miembros de la comunidad. Identifican también el alcance del uso de estrategias pedagógicas que faciliten la apropiación del manejo de las bases de datos. 
Finalmente, es clave resaltar que las diferentes áreas de la salud, incluida la Odontología, se encuentran en un proceso permanente de cambio, por lo que se hace necesaria la actualización teórica para un desarrollo profesional exitoso.

\section{Referencias}

1. Cabero-Almenara J. La formación virtual: principios, bases y preocupaciones La formación virtual: principios, bases y preocupaciones. 2000;83-102.

2. Hueso-Ibáñez L. Base de datos: grado superior [Internet]. Segunda. RA-MA. E, editor. Madrid; 2014.2 Available from: https://books.google.com.co/books?id= 12fDwAAQBAJ\&pg=PA9\&hl=es\&source=gbs toc $r \& c a d=3 \# v=o n e p a g e \& q \& f=f a l s e$

3. UNESCO. Conferencia Mundial sobre la Educación Superior-La educación Superior en el siglo XXI-Visión y acción. Compend la Declar Mund sobre la Educ Super. 1998;(Tomo I).

4. Organización de las Naciones Unidas para la Educación la C y la C. Conferencia Mundial sobre la Educación Superior - 2009: La nueva dinámica de la educación superior y la investigación para el cambio social y el desarrollo. UNESCO. 2009;81.

5. Beneitone P, González J, Wagenaar R. Informe Final Proyecto Tuning - América Latina: reflexiones y perspectivas de la Educación Superior en América Latina 2004-2007. Tuning. 2013.

6. Herazo-Acuña B, Hernandez G, Orlando-Cuéllar J, Gaidos C, Atuesta MF, CastellanosRobayo J, et al. Perfil y competencias profesionales del odontólogo en Colombia. Minist Salud y Protección Soc. 2013;1-46.

7. Ospina EG, Herault LR, Cardona AF. Uso de bases de datos bibliográficas por investigadores biomédicos latinoamericanos hispanoparlantes: Estudio transversal. Rev Panam Salud Publica/Pan Am J Public Heal. 2005;17(4):230-6.

8. Escolar R, Distritel E, Sede G, Escolar R, Distritel E, Sede G, et al. Enfoques teóricos sobre la, percepción que tienen las personas. Horiz Pedegóg. 2006;8(1):9-22.

9. Hernandez-Sampieri R, Fernandez-Collado C, Baptista-Lucio P. Metodología de la Investigación. Cuarta. McGraw-Hill, editor. México D.F.; 2006. 
10. Páramo-Bernal P. La clasificación múltiple de ítems (CMI). In: Colombia UP de, editor. La Investigación en Ciencias Sociales: Técnicas de recolección de la información. Bogotá; 2008.

11. Misas-Avella M. La educación líquida: aproximaciones a la realización sociedad-escuelasujeto en la determinación del proyecto de vida. Primera. Humanidades EA de, editor. Bogotá; 2015.

12. International Q. Nvivo ${ }^{\mathrm{TM}}$ [Internet]. 2020 [cited 2020 Oct 22]. Available from: https://www.qsrinternational.com/nvivo-qualitative-data-analysis-software/home

13. Morin E. Los siete saberes necesarios para la educación del futuro. Organ las Nac Unidas para la Educ la Cienc y la Cult. 1999;1-58.

14. Asenjo-Lobos C, Manterola C. Análisis Crítico de la Literatura. Un proceso elemental en el ejercicio de la práctica clínica basada en la evidencia. Int J Morphol. 2014;32(3): 9505.

15. Agudelo-Giraldo MA. Una aproximación a la consolidación de líneas de investigación desde la educación, la comunicación y la tecnología. Rev Electrónica la Red Investig Educ. 2004;1(1):1-8.

16. Díaz-Mendoza Y, Baena-Castro MA, Baena-Castro GR. Nuevos Escenarios De Aprendizaje, Un Reto Pedagógico. Cuad Educ y Desarro. 2018;(95):1-19.

17. Cóndor-Herrera O. Educar en tiempos de COVID-19. CienciAmérica. 2020;9(2).

18. Tisi Lanchares JP. Virtualización de los currículos odontológicos en tiempos de COVID19. Odovtos - Int J Dent Sci. 2020;(22):15-7.

19. Romanov K, Aarnio M. A survey of the use of electronic scientific information resources among medical and dental students. BMC Med Educ. 2006;6(28).

20. Barros-Bastidas C, Turpo-Gebera O. La formación en el desarrollo del docente investigador: Una revisión sistemática. Espacios. 2017;38(45).

21. Infante-Moro A, Torres-Díaz J, Infante-Moro J, Muñoz-Vázquez M. Evaluación del uso de las bases de datos electrónicas en la docencia universitaria de la Universidad de Huelva. RED Rev Educ a Distancia. 2014;(40):1-10. 
22. Franco-Cortés ÁM. La investigación formativa en la Facultad de Odontología de la Universidad de Antioquia: vivencias de los estudiantes de pregrado. Rev Nac Odontol. 2015;11(21).

23. Córdoba ME. Reflexión sobre la formación investigativa de los estudiantes de pregrado. Rev Virtual Univ Católica del Norte. 2016;47:20-37. 